\title{
MCP-1 and MIP-3 $\alpha$ Secreted from Necrotic Cell-Treated Glioblastoma Cells Promote Migration/Infiltration of Microglia
}

\author{
Yieun Jung ${ }^{a, b}$ So-Hee Ahn ${ }^{a, b}$ Hyunju Park ${ }^{a, b}$ Sang Hui Park ${ }^{c}$ Kyungsun Choid \\ Chulhee Choid,e Jihee Lee Kanga,b Youn-Hee Choi ${ }^{a, b}$
}

\begin{abstract}
aDepartment of physiology, Ewha Womans University School of medicine, Seoul, ${ }^{b} T$ issue Injury Defense Research Center, Ewha Womans University School of medicine, Seoul, 'Department of Pathology, Ewha Womans University School of Medicine, Seoul, 'Cellex Life Sciences Inc., Daejeon, Korea, eDepartment of Bio and Brain Engineering, KAIST, Daejeon, Korea
\end{abstract}

\section{Key Words}

Necrosis • Glioblastoma • Microglia • Migration • Infiltration

\begin{abstract}
Background/Aims: Glioblastoma multiforme (GBM) is the most common primary brain tumor in adults. The defining characteristics of GBM are diffuse infiltration of tumor cells into normal brain parenchyma, rapid growth, a high degree of infiltration of microglia and macrophages, and the presence of necrosis. Microglia/macrophages are frequently found in gliomas and they extensively infiltrate GBM tissue, up to $30 \%$ of total tumor mass. However, little is known about the effect of necrotic cells (NCs) on microglia infiltration in GBM and the tumor-infiltrating microglia-induced factors in GBMs. Methods: In this study, to address whether necrosis or necrosis-exposed GBM cells affect the degree of microglia/macrophage infiltration, migration and invasion/infiltration assays were performed. Culture supernatants and nuclear extracts of CRT-MG cells treated or untreated with necrotic cells were analyzed using a chemokine array and electrophoretic mobility shift assay, respectively. Results: The presence of NCs promoted the migration/infiltration of microglia, and GBM cell line CRT-MG cells exposed to NCs further enhanced the migration and infiltration of $\mathrm{HMO} 6$ microglial cells. Treatment with NCs induced mRNA and protein expression of chemokines such as Monocyte Chemoattractant Protein-1 (CCL2/MCP-1) and Macrophage Inflammatory Protein-3 $\alpha$ (CCL20/MIP-3 $\alpha$ ) in CRT-MG cells. In particular, CCL2/MCP-1 and CCL20/MIP-3 $\alpha$ were significantly increased in NC-treated CRT-MG cells. NCs induced DNA binding of the transcription factors Nuclear Factor (NF)- $\mathrm{KB}$ and Activator Protein 1 (AP-1) to the CCL2/MCP-1 and CCL20/MIP-3 $\alpha$ promoters, leading to increased CCL2/MCP-1 and CCL20/MIP-3 $\alpha$ mRNA and protein expression in CRT-MG cells. Conclusion: These results provide evidence that NCs induce the expression of CCL2/MCP-1 and CCL20/MIP-3 $\alpha$ in glioblastoma cells through activation of NF-KB and AP-1 and facilitate the infiltration of microglia into tumor tissues.

Yieun Jung and So-Hee Ahn contributed equally to this work




\section{Introduction}

Astrocytoma is the most prevalent form of primary brain cancer, originating from starshaped brain cells known as astrocytes [1]. Astrocytoma is divided into four pathologic grades by World Health Organization (WHO) classification; WHO grade IV glioblastoma multiforme (GBM) is the most malignant type of astrocytoma. GBM is a highly lethal cancer; patient survival with GBM is very poor, ranging from 12-15 months, despite aggressive treatment with surgery combined with chemotherapy and/or radiotherapy [2,3]. GBM is characterized by the presence of necrosis, microglia/macrophage infiltration, vascular proliferation, and diffuse invasion [4-6]. The presence of necrosis has been considered a key diagnostic criterion for GBM and is correlated with poorer patient outcome [7]. Some studies have suggested a direct correlation between the grade of astrocytoma and the levels of resident tumor microglia/macrophage by demonstrating a large number of microglia/macrophages in the necrotic areas [8, 9]. Microglia occupy 5-20\% of the total glial cell population, serving as the resident macrophages in the central nervous system (CNS) [10]. Microglia are critical effector cells of the immune response in the CNS [11]. They constantly destroy pathogens or remove dead cells and debris and also promote tissue repair and neural regeneration $[12,13]$. Recent evidence shows that the density of tumor-associated microglia/macrophage (TAM) in GBM is positively correlated with astrocytoma grade, proliferation, and invasiveness [9, $14,15]$. TAMs receive signals from diverse cells within the tumor microenvironment, which is regulated by several chemokines and chemokine receptors $[16,17]$.

Chemokines are a large group of small molecular weight proteins (8-12 kD) that interact with G protein-coupled transmembrane chemokine receptors. To date, more than 50 chemokines and 20 chemokine receptors have been identified [18]. Chemokines are classified into several structural groups (C, CC, CXC, and CX3C) based on the spacing of their cysteine residues [19]. Chemokines actively participate in inflammatory responses, such as allergies, autoimmune diseases, and viral infections, by attracting immune cells to the site of damaged and affected areas [20-22]. In addition, growing evidence indicates that chemokines play a crucial role in the development of cancer, promoting growth, angiogenesis, and metastasis. For example, Growth-regulated $\underline{\text { Oncogene }} \alpha$ (CXCL1/GRO $\alpha$ ) expression in hepatocellular carcinoma (HCC) led to increased HCC cell proliferation, growth, and invasion [23]. In

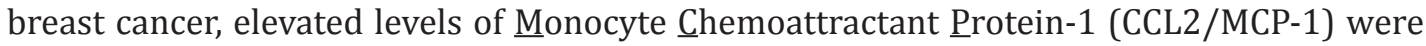
associated with macrophage accumulation and angiogenesis [24]. The effects of chemokines and chemokine receptors on cell migration and invasion have been demonstrated in several studies. Macrophage Inflammatory Protein-3 $\alpha$ (MIP-3 $\alpha / C C L 20)$ promotes the invasion and migration of thyroid cancer cells [25]. The interaction between CCL22 and CCR4 contributes to the migration of prostate cancer cells [26]. In our previous work, we showed that necrotic cells (NCs) induced secretion of the CXC motif chemokine ligand 8/interleukin-8 (CXCL8/ IL-8) in glioblastoma, and this influenced migration and invasion of glioblastoma cells [6].

Several chemokines have been suggested as potential factors that recruit macrophage/ microglia to tumors, including CCL2/MCP-1 and Regulated on Activation, Normal $\underline{T}$ cell Expressed and Secreted (CCL5/RANTES) [27]. Previous studies have shown that chemokines, such as CX3CL1/fractalkine and CCL2/MCP-1, and their receptors play crucial roles in the infiltration of microglia/macrophages in GBM $[17,28,29]$. To date, no study has characterized the effect of NCs or NC-treated GBM cells on tumor infiltration by microglia. In this study, to address whether necrosis or NC-treated GBM cells affect the degree of microglia/ macrophage infiltration and migration, invasion/infiltration assays were performed, and chemokine expression was profiled using microarray and a chemokine array.

\section{Materials and Methods}

\section{Cells}

Human glioblastoma cell line CRT-MG and U87-MG [30] and the immortalized human microglial cell line HMO6, which has been previously established [31] and was kindly provided by Prof. S.U. Kim, were 


\section{Cellular Physiology Cell Physiol Biochem 2018;48:1332-1346 \\ \begin{tabular}{l|l|l} 
and Biochemistry $10.1159 / 000492092$ & $\begin{array}{l}\text { D } 2018 \text { The Author(s). Published by S. Karger AG, Basel } \\
\text { www.karger.com/cpb }\end{array}$ \\
\hline
\end{tabular}}

Jung et al.: Necrosis Induces Microglia Infiltration in Glioblastoma

maintained in Dulbecco's modified essential medium (DMEM, WelGENE Inc., Daegu, Korea) containing 10\% fetal bovine serum (FBS, WelGENE Inc.), L-glutamine, $100 \mathrm{U} / \mathrm{ml}$ penicillin, and $10 \mu \mathrm{g} / \mathrm{ml}$ streptomycin. For preparation of necrotic cells, CRT-MG and U87-MG cells were frozen and thawed through five cycles of liquid nitrogen-water bath treatment as described previously [6].

\section{Reagents and antibodies}

Human recombinant CCL2/MCP-1, CCL20/MIP-3 $\alpha$ and CXCL8/IL-8 proteins, and polyclonal antihuman CCL2/MCP-1, CCL20/MIP-3 $\alpha$ and CXCL8/IL-8 antibodies were obtained from R\&D Systems (Minneapolis, MN, USA). Control nonspecific IgG from goat serum was purchased from Sigma-Aldrich, Co.

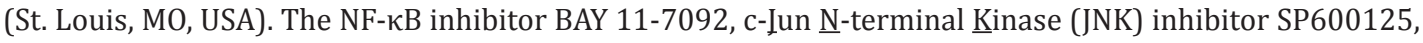
p38 inhibitor SB203580 and were purchased from Sigma-Aldrich, Co. Extracellular signal-regulated Kinase (ERK) inhibitor PD98059 was obtained from Merck (Darmstadt, Germany). Exo-mCherry (mChrry-loaded exosome) and Exо-srIкB (srIкB-loaded exosome) were obtained from Cellex Life Sciences Inc. (Daejeon, Korea) [32].

\section{Cell migration assay}

The ability of HMO6 cells to migrate through membrane was measured in a Boyden chamber. HMO6 cells $\left(1.5 \times 10^{5}\right)$ suspended in $1 \%$ FBS culture media placed in the top chamber of $8.0 \mu$ m polycarbonate membrane transwell inserts (Corning Inc., Corning, NY, USA). CRT-MG cells were either untreated or treated with necrotic CRT-MG cells for $24 \mathrm{~h}$, and then supernatants were collected and centrifuged at $4{ }^{\circ} \mathrm{C}, 12,000$ rpm $(13523 \mathrm{~g}$ ) for $20 \mathrm{~min}$. The culture supernatants of CRT-MG either untreated or treated with necrotic cells and necrotic cell were placed in the lower chamber (Corning Inc.) as a chemoattractant. After $24 \mathrm{~h}$, cells that migrated through the pores were collected and centrifuged, and then living cells were counted using a hemocytometer (Superior, Marienfeld, Germany) after trypan blue staining.

\section{Cell invasion/infiltration assay}

For invasion/infiltration assay, the upper surface membranes of transwell inserts (Corning Inc.) were coated with $200 \mu \mathrm{l}$ matrigel plus cold serum-free DMEM allowed to solidify overnight at $37{ }^{\circ} \mathrm{C}$. HMO6 cells were seeded onto the upper chambers. The lower chamber contained either necrotic cell lysates or the supernatants obtained from necrotic cells-treated or untreated CRT-MG cells. After $24 \mathrm{~h}$ incubation, noninvaded cells in the upper chambers were removed with a cotton swab, and invaded cells were fixed in $4 \%$ formaldehyde for $10 \mathrm{~min}$ and washed with phosphate-buffered saline (PBS). Fixed cells were stained with $0.1 \%$ crystal violet for 10 min and then washed with PBS. After being dried, the stained cells were observed using Eclipse TE-300 microscope (Nikon, Tokyo, Japan). For quantification, crystal violet staining was resolubilized in 95\% ethanol and the optical density (O.D.) at $540 \mathrm{~nm}$ was measured.

\section{Microarray analysis}

Microarray analysis was performed using Illumina Human HT-12 B4 Expression BeadChip (Illumina, Inc., San Diego, USA) according to the manufacturer's protocol at Macrogen (Macrogen, Seoul, Korea). The array detects the expression levels for more than 47, 000 probes derived from the National Center for Biotechnology Information Reference Sequence (NCBI) RefSeu Release 38. Total RNA was extracted from the cells using a RNA extract kit (easy-Blue ${ }^{\mathrm{TM}}$, Intron, Gyeonggido, Korea) and 500 ng of total RNA from each sample amplified and labelled using TargetAmp-Nano Labeling Kit for Illumina Expression BeadChip (Epicentre, Madison, USA). 750 ng of labeled cRNA were hybridized to the Human HT-12 V4.0 Expression Beadchip for $17 \mathrm{~h}$ at $58^{\circ} \mathrm{C}$ Arrays were scanned using the Illumina Bead Array Reader Confocal Scanner and data were extracted using GenomeStudio v2011.1 (Gene Expression Module v1.9.0).

\section{Human chemokine array}

Chemokines secreted by necrotic cells-treated CRT-MG were screened using commercially available protein array systems (Proteome profiler ${ }^{\mathrm{TM}}$ Arrays, \#ARY017, R\&D Systems) according to the manufacturer's instructions. The array consists of 31 different human chemokines spotted in duplicate onto a membrane. CRT-MG cells were either untreated or treated with necrotic cells for $24 \mathrm{~h}$, and then $1 \mathrm{ml}$ of the supernatant and detection antibody mixture were incubated at room temperature (RT) for $1 \mathrm{~h}$. Then, the prepared mixtures were added on each membrane at $4^{\circ} \mathrm{C}$ for overnight, and streptavidin-HRP was treated into each 


\section{Cellular Physiology Cell Physiol Biochem 2018;48:1332-1346

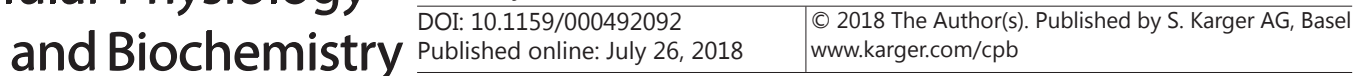

Jung et al.: Necrosis Induces Microglia Infiltration in Glioblastoma

membrane for $30 \mathrm{~min}$ at RT on a rocking platform shaker. Membranes were exposed to blue X-ray film (AGFA, Mortsel, Belgium) by using chemiluminescent detection reagent (Amersham, Buckinghamshire, UK) for 3-5 min. The relative level of chemokine expression was measured by the changed spot intensity divided by control spot intensity, using Image J software (NIH, Bethesda, Maryland, USA; http://rsb. info.nih.gov/ $\mathrm{ij} /)$.

Reverse transcription-polymerase chain reaction (RT-PCR) and quantitative RT-PCR (qRT-PCR)

Total cellular RNA was extracted from isolated HMO6 cells using easy-BLUE ${ }^{\mathrm{TM}}$ (Intron) according to the manufacturer's protocol. cDNA was synthesized from $1 \mu \mathrm{g}$ of total RNA with reverse transcription and PCR was performed using $3 \mu \mathrm{l}$ cDNA with a thermal cycler (Bio-Rad Laboratories, Hercules, CA, USA). Quantitative PCR was performed on an ABI StepOnePlus ${ }^{\mathrm{TM}}$ Real-Time PCR machine (Applied Biosystems, Foster City, CA, USA) using a Power SYBR Green PCR Master Mix (Applied Biosystems) according to the manufacturer's protocol. The cDNA abundance of each gene was calculated relative to the expression of a housekeeping gene, GAPDH (glyceraldehyde-3-phosphate-dehydrogenase) and presented as the foldchange in abundance compared to the appropriate controls. The primer sequences were as follows: CXCL8/IL-8, forward 5'-CTCCAAACCTTTCCACCCC-3', reverse 5'-GATTCTTGGATACCACAGAGAATG-3'; CCL2/MCP-1, forward 5'-CCCCA GTCACCTGCTGTTAT-3', reverse 5'-TGGAATCCTGAACCCACTTC-3'; CCL20/MIP-3 $\alpha$, forward 5'-GCAAGCAACTTTGACTGCTG-3', reverse 5'-ATTTGCGCACACACAGACAA CT3'; CCR2, forward 5'-AGAGGCATAGGGCAGTGAGA-3', reverse 5'-GCAGTGAGTC ATCCCAAGAG-3'; CCR6, forward 5'-CCATTCTGGGCAGTGAGTCA-3', reverse 5'-AG CAGCATCCCGCAGTTAA-3'; GAPDH, forward 5'-GGAGCCAAAAGGGTCATCAT-3', reverse 5'-GTGATGGCATGGACTGTGGT-3'.

\section{Enzyme-linked immunosorbent assay (ELISA)}

Chemokine levels were measured in the culture supernatants of CRT-MG plated in $60 \mathrm{~mm}$ culture plates at final concentration of $9 \times 10^{5}$ cells. After stabilization, cells were treated with either untreated or treated with necrotic cells for $24 \mathrm{~h}$. Supernatants were obtained and centrifuged at $4^{\circ} \mathrm{C}, 12,000 \mathrm{rpm}(13523 \mathrm{~g})$ for $20 \mathrm{~min}$ and chemokine concentrations were determined by a sandwich ELISA method with human CCL2/ MCP-1 and CCL20/MIP-3 $\alpha$ (R\&D Systems) according to the manufacturer's instructions. The concentration of CCL2/MCP-1 and CCL20/MIP-3 $\alpha$ in each sample was determined with a standard curve generated by known amounts of CCL2/MCP-1 and CCL20/MIP-3 $\alpha$.

\section{Electrophoretic mobility shift assays (EMSA)}

EMSA was performed with $3 \mu \mathrm{g}$ of nuclear extracts, as previously described [6]. Briefly, nuclear extracts from CRT-MG treated with or without necrotic cells were incubated with the CCL2/MCP-1 or CCL20/MIP-3 $\alpha$ sequences including putative NF- $\kappa \mathrm{B}$ or AP-1 binding element, which was end-labeled with [32P] ATP using T4 polynucleotide kinase (New England Biolabs, Inc (NEB), Ipswich, MA, USA). Bound and free DNA were resolved by electrophoresis in 5\% native polyacrylamide gels using $0.5 \mathrm{X}$ Tris-borate-EDTA (TBE) as running buffer. The gels were dried and then bound DNA was assessed by autography. Competition assays were performed with 100-fold molar excess of unlabeled oligonucleotide was added to the nuclear extracts for 10 min before addition of the labeled probe. For supershift experiments, binding reactions containing nuclear extract were incubated for $30 \mathrm{~min}$ at RT with anti-c-Fos, anti-p50 or anti-p65 antibody (1/100 dilution), (Santa Cruz, CA, USA). The oligonucleotide probe sequences and putative NF- $\kappa$ B or AP-1 binding sites are shown in Fig. 4.

\section{Statistical analysis}

Mean \pm standard error of the mean (SEM) were showed in all bar graphs from at least three independent experiments, and statistical significance was analyzed by ANOVA and student t-test using the SPSS software version 23.0 (SPSS Inc., Chicago, IL, USA). A value of $p<0.05$ was regarded statistically significant.

\section{Results}

NCs and NC-treated glioma cells induce migration and infiltration of microglial cells

We first investigated the effect of 1) necrotic CRT-MG cells and 2) CRT-MG cells exposed to the necrotic CRT-MG cells (NC-treated CRT-MG) on the migration and infiltration of HMO6 
human microglial cells. Necrotic CRT-MG cells were prepared by freezing/thawing CRT-MG cells for five cycles. Next, different ratios of necrotic CRT-MG cells were either diluted in media (NC-only) or used to treat pre-seeded CRT-MG cells for $24 \mathrm{~h}$. Media were collected from the pre-seeded CRT-MG culture (NC-treated glioblastoma conditioned medium, NCGCM) and the NC-only group. HMO6 cells were seeded at an equal density $\left(1.5 \times 10^{5}\right.$ cells/ well) in the upper chamber, and the collected NC-GCM and NC-only were placed in the lower chambers (Fig. 1a). As shown in Fig. 1b, both NC-GCM and NC-only stimulation increased the migration of HMO6 cells. To further explore the effect of NC-only and NC-GCM on HMO6 cell infiltration, matrigel matrix-coated 24-well microchemotaxis chambers were used in the presence of the NC-GCM or NC-only culture supernatants in the lower chambers. As shown in Fig. 1c, NC-GCM and NC-only increased HMO6 cell infiltration in a ratio-dependent manner. Interestingly, the migration and infiltration activity of HMO6 cells showed a tendency toward a significant increase in NC-GCM compared to NC-only, suggesting that CRT-MG cells exposed to NCs enhance the migration and infiltration of HMO6 microglial cells.

Effect of NCS on gene and chemokine expression in CRT-MG glioma cells

We next performed a microarray to identify the changes in gene expression profiles between CRT-MG cells (Con) and NC-treated CRT-MG cells (NC) (Fig. 2a). For this analysis, CRT-MG cells were either treated with NCs or not treated for $24 \mathrm{~h}$, and purified RNA was prepared for each group. Of 47, 323 genes detected by the array, 46, 580 genes were not significantly influenced by the exposure to NCs. In the treated cells, 439 genes were upregulated, and 304 genes were downregulated (a 2-fold change was used as the cut off). Compared with untreated cells, dysregulated expression levels with 2-fold change in NCtreated CRT-MG cells were tabulated (Fig. 2b). CCL20/MIP-3 $\alpha$, CXCL8/IL-8, CXCL2/MIP$2 \alpha$, CXC motif chemokine ligand 5/Epithelial cell-derived Neutrophil-activating peptide 78 (CXCL5/ENA-78), CCL2/MCP-1, and CX3CL1/fractalkine were significantly increased in NC-treated CRT-MG cells. We also utilized reverse transcription-polymerase chain reaction (RT-PCR) and quantitative real-time PCR (qRT-PCR) for direct validation of differential expression of mRNA in necrotic cell treated (NC) and untreated CRT-MG cells (Con) (Fig. 2c). To examine whether NCs induced chemokine secretion in CRT-MG cells, we performed a chemokine array with the culture media from CRT-MG that were either treated with NCs for $24 \mathrm{~h}$ or untreated. The chemokine array showed that the secretion of several chemokines was significantly increased in NC-treated CRT-MG cells compared to that in the control (Fig. $2 \mathrm{~d}$, solid box). These results clearly show that NCs induce the expression and secretion of several inflammatory chemokines such as CCL2/MCP-1 and CCL20/MIP-3 $\alpha$ in CRT-MG glioma cells.

NCs induce protein and mRNA expression of CCL2/MCP-1 and CCL20/MIP-3 $\alpha$ in CRT-MG glioma cells

Since we observed that the fold intensity changes for spots corresponding to CCL2/ MCP-1 and CCL20/MIP-3 $\alpha$ were increased over 100-fold by exposure to necrotic cells (Fig. 2d), we next performed ELISA to detect and quantify CCL2/MCP-1 and CCL20/MIP-3 $\alpha$ levels secreted from NC-treated CRT-MG cells. MCP-1 and MIP-3 $\alpha$ secretion was increased in both CRT-MG cells treated with NC and NC-only in a ratio-dependent manner; however, the degree of secretion of MCP- 1 and MIP-3 $\alpha$ was higher in CRT-MG cells treated with NC than in NC-only. (Figs. 3b and 3c). Similar to protein expression, expression of CCL2/MCP-1 and CCL20/MIP$3 \alpha$ mRNA was also increased in NC-treated CRT-MG cells (Figs. 3d and 3e). Similar effects of necrotic cells on CCL2/MCP-1 and CCL20/MIP-3 $\alpha$ induction were also observed in U87MG cells (Figs. $3 \mathrm{f}$ and $3 \mathrm{~g}$ ). These results demonstrate that NCs directly induce expression of CCL2/MCP-1 and CCL20/MIP-3 $\alpha$ in glioma cells.

NCs induce NF- $\kappa B$ and AP-1 DNA binding to the CCL2/MCP-1 and CCL20/MIP-3 $\alpha$ promoters in CRT-MG glioma cells

Recently we reported that NCs induce the activation of AP-1 and NF- $\mathrm{BB}$ signaling pathways, leading to enhanced DNA binding to the IL-8 promoter [6]. To investigate the 


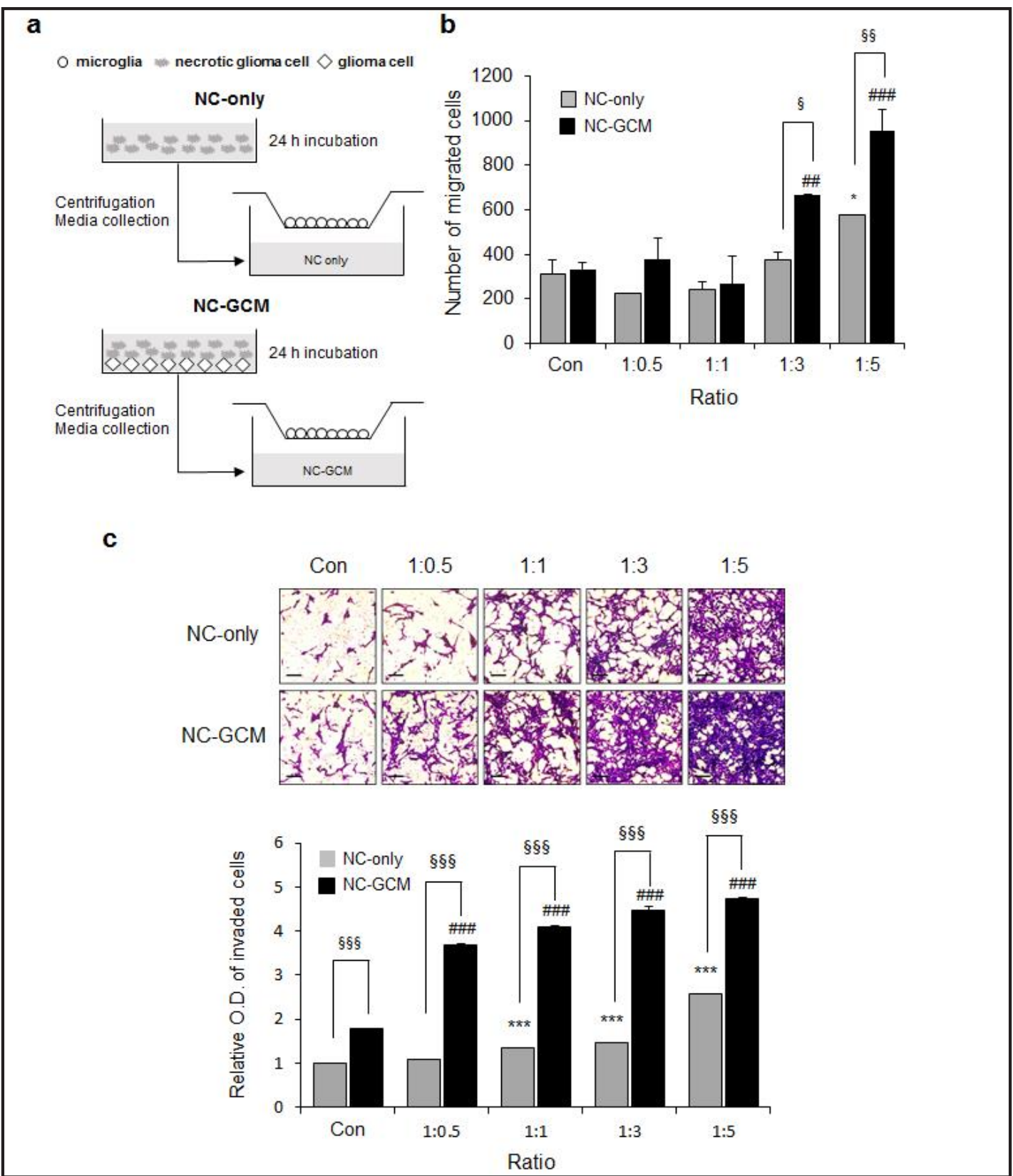

Fig. 1. Necrotic cell (NC)-treated glioma cells induce migration and infiltration of microglia. (a) Illustration of the transwell migration assay. CRT-MG cells treated with different ratios of necrotic cell for $24 \mathrm{~h}$ as indicated, and each NC-treated glioblastoma conditioned medium (NC-GCM) and necrotic cells conditioned medium (NC-only) were collected and centrifugation. HMO6 cells were seeded at equal density $\left(1.5 \times 10^{5}\right.$ cells/well) in the upper chamber, and the collected NC-GCM and NC-only were placed in the lower chambers. The ratio of NC treatment is shown as CRT-MG cell number: necrotic CRT-MG cell number. (b) Migration of HMO6 cell was determined by counting cells that migrated through the membrane of transwell inserts. Data shown

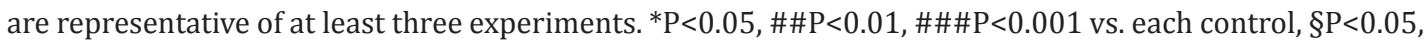
$\S \S \mathrm{P}<0.01$ vs NC-only. (c) Infiltration of HMO6 cell was assayed with a matrigel matrix-coated cell culture insert. Representative images of crystal violet staining of the bottom surface of cell culture insert are shown (upper panel, scale bar $=100 \mu \mathrm{m}$ ). Cell infiltration was measured by OD quantification by reading the eluted crystal violet (bottom panel). The extent of infiltration in the NC-only control was set 1 and data presented as the fold induction compared with NC-only control cells. Data shown are representative of at least three experiments. ${ }^{* * *} \mathrm{P}<0.001, \# \# \# \mathrm{P}<0.001$ vs. each control (Con), $\S \S \S \mathrm{P}<0.01$ vs. NC-only. 
Fig. 2. Effect of necrotic cells on chemokine expression in CRT-MG glioma cells. (a) CRT-MG cells treated with necrotic cell and untreated cells for $24 \mathrm{~h}$ as indicated. After incubation, cells and supernatants from each condition were collected for microarray and chemokine array respectively. Con, control; NC, necrotic cells. (b) CRT-MG cells were incubated in the absence or presence of NCs for $24 \mathrm{~h}$, and total RNA was extracted and subjected to microarray analysis. The proportion of genes that are up- or downregulated (2-fold change) between untreated and NC-treated cells is shown. Tabulation of upregulated chemokine genes demonstrating higher fold induction in NC-treated CRT-MG cells compared with that in untreated CRTMG cells. (c) The expression level of three upregulated mRNA (CCL2/MCP-1, CCL20/ MIP-3 $\alpha$ and CXCL8/IL-8) were validated by RT-PCR and qRT-PCR. GAPDH gene was evaluated as external control. The expression of each mRNA in the untreated control was set as 1. Data shown are representative of at least three experiments.

$* * * \mathrm{P}<0.001$ vs. Con (untreated control). (d) Culture supernatants of CRT-MG cells treated or untreated with NCs for $24 \mathrm{~h}$ were analyzed using a chemokine array. A solid box indicates the duplicate spots corresponding to six chemokines, and reference spots are indicated by a dashed line box (left). The intensity of spots corresponding to each chemokine and positive control was quantified using Image J software and subtracted from the background, then expressed as a ratio to the positive control (right). The value of each chemokine in the untreated control was set as 1 . Data are representative of at least 3 experiments. ${ }^{* *} \mathrm{P}<0.01,{ }^{* * *} \mathrm{P}<0.001$ vs. Con (untreated control).

contribution of AP-1 and NF- $\kappa$ B to NC-induced CCL2/MCP-1 and CCL20/MIP-3 $\alpha$ expression, we next performed an electrophoretic mobility shift assay (EMSA). We found putative NF- $\kappa \mathrm{B}$ and AP-1 transcription factor-binding sites in the CCL2/MCP-1 and CCL20/MIP-3 $\alpha$ promoters (Figs. 4a and 4b). Nuclear extracts (NEs) from untreated or different ratios of NCs-treated CRT-MG cells were analyzed with probes for putative binding sites within the CCL2/MCP-1 or CCL20/MIP-3 $\alpha$ promoters (Figs. 4c and 4d). Utilizing a DNA probe containing the specific NF- $\kappa B$ binding site (2) of the CCL2/MCP-1 promoter, binding was increased 


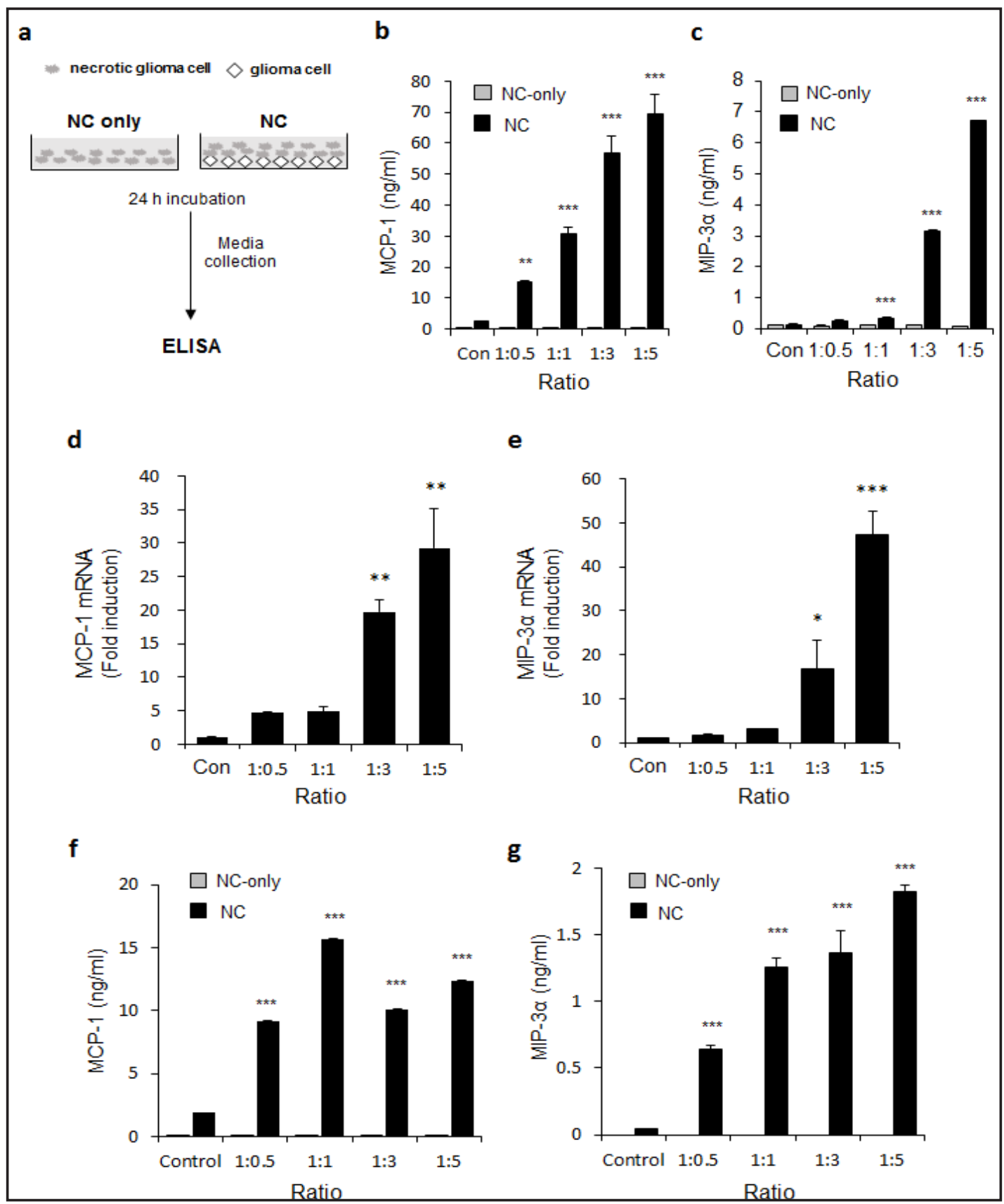

Fig. 3. Necrotic cells induce mRNA and protein expression of CCL2/MCP-1 and CCL20/MIP-3 $\alpha$ in glioma cells. (a) CRT-MG cells treated with different ratios of necrotic cells (NC) for $24 \mathrm{~h}$ as indicated. After incubation, supernatants from each condition and NC-diluted medium (NC-only) were collected, and secretory protein levels were measured by ELISA. (b, c) The protein levels from the culture supernatants were analyzed via ELISA for CCL2/MCP-1 (b) and CCL20/MIP-3 $\alpha$ (c). Data shown are representative of at least three experiments. ${ }^{* *} \mathrm{P}<0.01,{ }^{* * *} \mathrm{P}<0.001$ vs. Con (untreated control). (d, e) CRT-MG cells treated with different ratios of NCs for $24 \mathrm{~h}$ as indicated. After incubation, cells were collected, and total RNA was isolated using an RNA extraction kit. CCL2/MCP-1 and CCL20/MIP-3 $\alpha$ mRNA expression was analyzed by quantitative real-time PCR (qRT-PCR). (f, g) U87-MG cells treated with different ratios of necrotic cells (NC) for $24 \mathrm{~h}$. After incubation, supernatants from each condition and NC-diluted medium (NC-only) were collected and analyzed via ELISA for CCL2/MCP-1 (f) and CCL20/MIP-3 $\alpha$ (g). Data were presented as fold induction compared with untreated control cells. Data shown are representative of at least three independent experiments. ${ }^{*} \mathrm{P}<0.05,{ }^{* *} \mathrm{P}<0.01,{ }^{* * *} \mathrm{P}<0.001$ vs. Con (untreated control). 
in NC-treated CRT-MG cells in a ratio-dependent manner. In the CCL20/MIP-3 $\alpha$ promoter, DNA probes containing the specific NF- $\mathrm{KB}$ binding site (2) and AP-1 binding site (2) showed enhanced binding to NE from NC-treated CRT-MG. Competition using a 100-molar excess unlabeled oligonucleotide abrogated complex formation; in addition, antibodies for p50 or $\mathrm{c}$-fos supershifted and weakened the complex, confirming the specific interaction of NF- $\kappa \mathrm{B}$ and AP-1 in both CCL2/MCP-1 and CCL20/MIP-3 $\alpha$ promoters (Figs. 4e and 4f). These results indicate that NCs induce the activation of NF- $\mathrm{KB}$ and AP-1 transcription factors, leading to increased DNA binding activity to the CCL2/MCP-1 and CCL20/MIP-3 $\alpha$ promoters.

Inhibition of NF- $\kappa B$ and AP-1 reduces CCL2/MCP-1 and CCL20/MIP-3 $\alpha$ secretion in NCtreated CRT-MG cells

Since we observed that NF- $\mathrm{KB}$ and AP-1 were involved in regulation of CCL2/MCP-1 and CCL20/MIP-3 $\alpha$ genes in response to NC exposure (Fig. 4), we next examined the effect of inhibition of NF- $\mathrm{kB}, \mathrm{c}$-Jun N-terminal kinase (JNK), p38, and extracellular signal-regulated kinase (ERK) on NC-induced CCL2/MCP-1 and CCL20/MIP-3 $\alpha$ expression in glioma cells, using specific inhibitors for NF-кB: BAY 11-7082, JNK: SP600125, p38: SB203580 and ERK: PD98059. CRT-MG cells were pretreated with pharmacological inhibitors for $30 \mathrm{~min}$ and then exposed to NCs for 24 h. Both CCL2/MCP-1 and CCL20/MIP-3 $\alpha$ expression/secretion in NC-treated CRT-MG cells were most strongly suppressed by NF- $\kappa B$ inhibitor BAY 117082 (Figs. 5a and 5b). These results showed that NF- $\kappa B$ was to be major transcription factor that regulated CCL2/MCP-1 and CCL20/MIP-3 $\alpha$ expression. When CRT-MG cells were

Fig. 4. Necrotic cells increase NF-кB and AP-1 DNA binding to CCL2/MCP-1 and CCL20/MIP$3 \alpha$ promoters in CRT-MG glioma cells. Schematic representations of the human CCL2/MCP-1 (a) and CCL20/MIP-3 $\alpha$ (b) promoters. The transcription start site and putative binding sites for NF-кB and AP-1 are indicated by an arrow (upper), and details for each transcription factor are displayed in the table (lower). (c, d) The nuclear extracts (NEs) from CRT-MG cells treated with or without NCs were incubated with a radiolabeled DNA probe for the human CCL2/ MCP-1 (c) and CCL20/MIP-3 $\alpha$ (d) promoters and subjected to an electromobility shift assay (EMSA). The competition assay was performed by adding a 100-fold molar excess of cold probe. Data shown are representative of at least three experiments. (e,f) EMSA was performed using the labeled probes NF-КB (2) and AP-1 (2) for the CCL2/MCP-1 (e) or NF-кB (2) and AP-1 (2) for the CCL20/MIP-3 $\alpha$ (f) promoter. Supershift assays were performed with specific antibodies against NF-кB subunits p50 and p65 or the AP-1 subunit c-fos. Anti-p50, anti-p65, or anti-c-fos antibodies were added to test the specificity of interaction. Data shown are representative of at least three experiments. FP, free probe; C, competition.

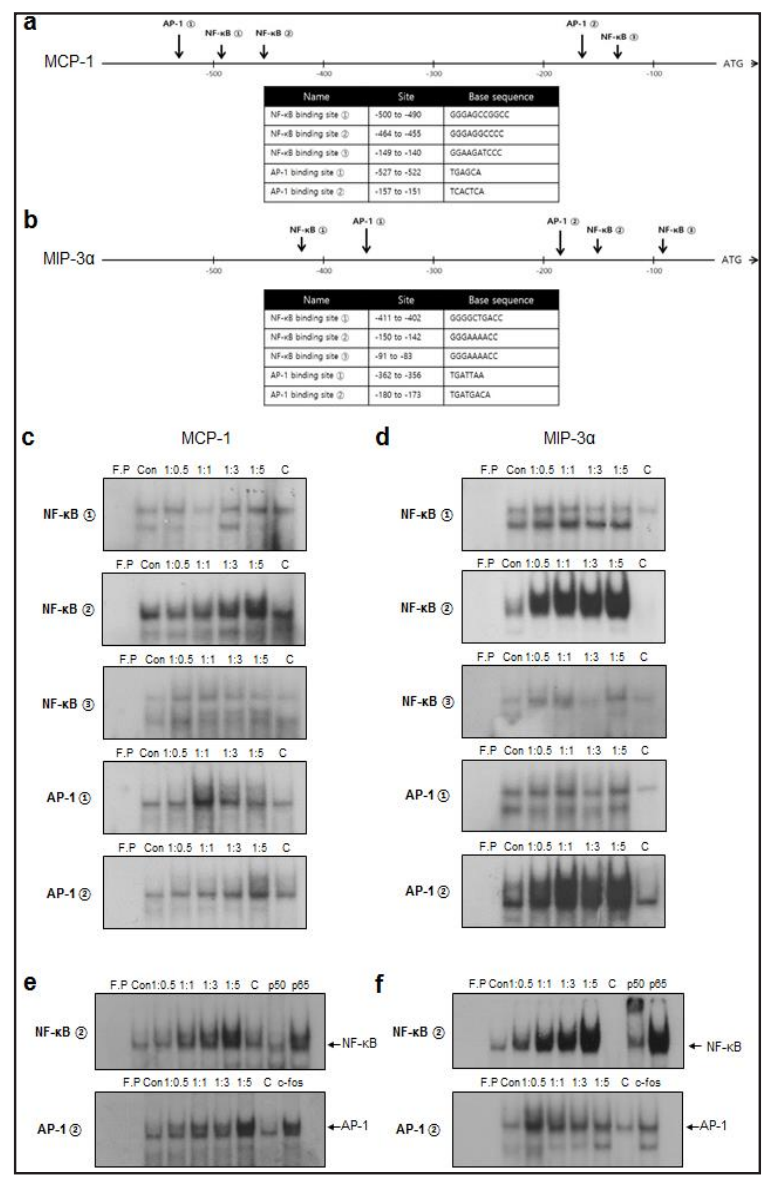


incubated with $5 \times 10^{8}$ particles $/ \mathrm{ml}$ mCherry:EXPLORs or srIkB:EXPLORs, srlkB-loaded exosome as a specific inhibitor for NF- $\kappa \mathrm{B}$, for $16 \mathrm{~h}$ and either treated with NCs or untreated for an additional $24 \mathrm{~h}$, we observed that treatment with srIкB:EXPLORs significantly reduced both CCL2/MCP1 and CCL20/MIP-3 $\alpha$ expression in NC-treated CRT-MG cells (Figs. 5c and $5 \mathrm{~d})$. These results collectively indicate that blocking NF- $\kappa$ B signaling pathways effectively reduces CCL2/MCP-1 and CCL20/MIP-3 $\alpha$ expression/secretion in CRT-MG glioma cells stimulated by NCs.

CCL2/MCP-1 and CCL20/MIP-3 $\alpha$ induce migration and infiltration of microglial cells

CCL2/MCP-1 and CCL20/MIP-3 $\alpha$ are generally considered important chemoattractants for monocytes [28]. Because NC-GCM induced both migration and infiltration of microglial cells (Fig. 1), and NC directly induced CCL2/MCP-1 and CCL20/MIP-3 $\alpha$ secretion in glioma cells, we asked whether CCL2/MCP-1 and CCL20/MIP$3 \alpha$ secreted from NC-treated glioma cells directly mediated the migration and infiltratin of HMO6 microglial cells. First, we investigated whether the receptors for CCL2/MCP-1 (CCR2) and CCL20/MIP-3 $\alpha$ (CCR6) are present in HMO6 microglial cells and whether NConly and NC-GCM affect the expression of CCR2 and CCR6 on microglia. HMO6 cells were 1) not treated or incubated with 2) glioblastoma conditioned media (GCM), 3) NC-only, or 4) NC-GCM for 24 h (Fig. 6a), and total RNA was extracted and analyzed by RT-PCR. CCL2/MCP1 receptor CCR2 and CCL20/MIP-3 $\alpha$ receptor CCR6 mRNA was expressed in HMO6 cells (Fig. 6b), similar to previous reports [33]. Previously, we showed that NCs induced the secretion of CXCL8/IL-8, which influenced the migration and invasion of glioblastoma cells. To test whether CCL2/MCP1, CCL20/MIP-3 $\alpha$, and CXCL8/IL-8 have effects on microglia migration/ infiltration, HMO6 cells were seeded at equal density $\left(1.5 \times 10^{5}\right.$ cells/well $)$ in the upper chamber, and control medium supplemented with $1 \mu \mathrm{g} / \mathrm{ml}$ of recombinant CCL2/MCP-1,

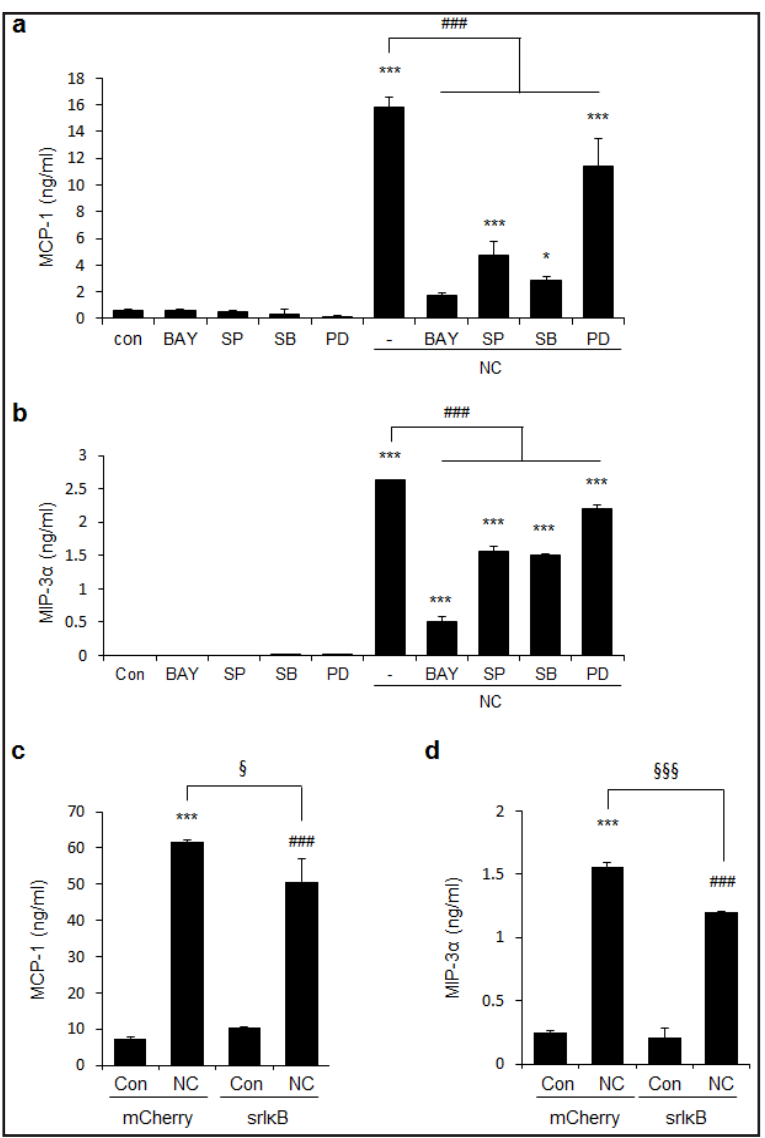

Fig. 5. Inhibition of NF-кB and AP-1 reduces CCL2/ MCP-1 and CCL20/MIP-3 $\alpha$ expression/secretion in CRT-MG cells. (a, b) CRT-MG cells were pretreated with NF- $\kappa B$ inhibitor (BAY 11-7082 [BAY]), a JNK inhibitor (SP600125 [SP]), a p38 inhibitor (SB203580 [SB]), or MAPK/ERK kinase inhibitor (PD98059 [PD]) $5 \mu \mathrm{M}$ for 30 min and then further incubated with necrotic cells (NC) for $24 \mathrm{~h}$. Indicated supernatants from each condition were collected, and then CCL2/MCP-1 (a) or CCL20/MIP-3 $\alpha$ (b) protein levels were measured by ELISA. Absorbance was measured at $450 \mathrm{~nm}$. Data shown represent three independent experiments. ${ }^{* * *} \mathrm{P}<0.001$ vs. Con, ${ }^{\# \# \# P<0.001}$ vs. NC. Con, untreated control; NC, necrotic cells; BAY, BAY11-7082; SP, SB600125; SB, SB203580; PD, PD98059. (c,d) CRT-MG cells were incubated with $5 \times 10^{8}$ particles/ $\mathrm{ml}$ mCherry:EXPLORs or srIкB:EXPLORs for $16 \mathrm{~h}$, and then treated with NCs for an additional $24 \mathrm{~h}$. Indicated supernatants from each condition were collected, and CCL2/MCP-1 (c) or CCL20/MIP-3 $\alpha$ (d) protein levels were measured by ELISA. Data shown represent three independent experiments. ${ }^{* * *} \mathrm{P}<0.001$ vs. mCherry Con, \#\#\#P<0.001 vs. srIкB Con, $\S \mathrm{P}<0.05$, $\S \S \mathrm{P}<0.05$ vs. mCherry NC. Con, untreated control; NC, necrotic cells. 
Fig. 6. Neutralizing antibodies to CCL2/MCP-1 and CCL20/MIP$3 \alpha$ block microglia infiltrationpromoting effects of necrotic celltreated glioblastoma conditioned medium. (a) Control medium (Con), glioblastoma conditioned medium (GCM), NC only, and NC-GCM were harvested from cultured supernatants for $24 \mathrm{~h}$ and treated to microglia cultures for $24 \mathrm{~h}$. (b) HMO6 microglia cells were either not treated or treated with GCM, NC-only, or NC-GCM for $24 \mathrm{~h}$, and total RNA was extracted and analyzed by RT-PCR for CCR2 and CCR6 mRNA. (c-f) Transwell migration and invasion/infiltration assay showing the migration and infiltration of HMO6 cells toward control medium supplemented with recombinant protein and NCGCM in the presence or absence neutralizing antibody. (c,d) The migration assay was performed using $1 \mu \mathrm{g} / \mathrm{ml}$ recombinant CCL2/ MCP-1, CCL20/MIP-3 $\alpha$, or CXCL8/ IL-8 protein (c). CRT-MG cells were either untreated or treated

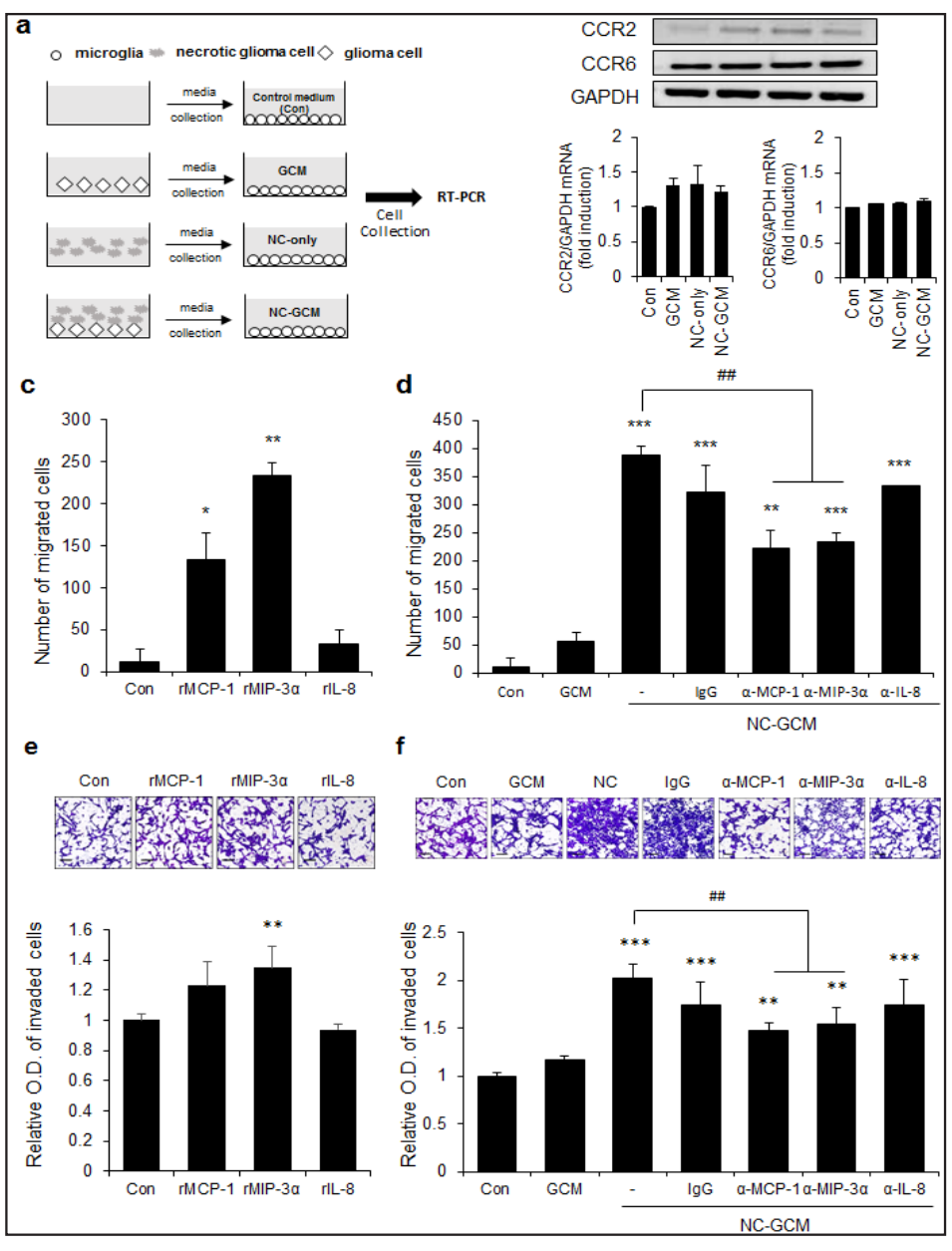
with NC for $24 \mathrm{~h}$ and collected.

NC-GCM was placed in the lower chamber in the presence or absence of $2 \mu \mathrm{g} / \mathrm{ml}$ anti-CCL2/MCP-1, antiCCL20/MIP-3 $\alpha$, or anti-CXCL8/IL-8 neutralizing antibody or nonspecific goat-IgG as a control (d). Data are representative of at least 3 experiments. ${ }^{*} \mathrm{P}<0.05$, ${ }^{* *} \mathrm{P}<0.01$, ${ }^{* * *} \mathrm{P}<0.001$ vs. Con, ${ }^{\# \#} \mathrm{P}<0.01$ vs. NC-GCM (-). (e, f) Invasion/infiltration of HMO6 cells was analyzed invasion/infiltration assay. Representative images of crystal violet staining of the bottom surface are shown (upper panel, scale bar $=100 \mu \mathrm{m}$ ). Cell infiltration was measured by OD quantification by reading the eluted crystal violet (bottom panel). The extent of infiltration in the control was set 1 and data presented as the fold induction compared with control. Data shown are representative of at least three experiments. ${ }^{*} \mathrm{P}<0.05,{ }^{* *} \mathrm{P}<0.01,{ }^{* * *} \mathrm{P}<0.001$ vs. Con, ${ }^{\# \#} \mathrm{P}<0.01$ vs. NC-GCM. Con, control medium; GCM, glioblastoma conditioned medium; NC, necrotic cells; NC-GCM, NCtreated glioblastoma conditioned medium; r, recombinant.

CCL20/MIP-3 $\alpha$, or CXCL8/IL-8 protein was placed in the lower chamber (Figs. 6c and 6e). In the presence of recombinant CCL2/MCP-1 and CCL20/MIP-3 $\alpha$, HMO6 migration and infiltration were increased, whereas the effect of CXCL8/IL-8 protein was not significant. Also, we investigated the migration and infiltration of HMO6 by incubating the NC-GCM with neutralizing antibodies for CCL2/MCP-1, CCL20/MIP-3 $\alpha$, or CXCL8/IL-8 (2 $\mu \mathrm{g} / \mathrm{ml})$. As shown in Figures 6d and 6f, anti-CCL2/MCP-1 and anti-CCL20/MIP-3 $\alpha$, but not anti-CXCL8/IL-8 and control non-specific IgG, significantly reduced the migration and infiltration of HMO6 cells. These results collectively indicate that CCL2/MCP-1 and CCL20/MIP-3 $\alpha$, which are secreted from NC-treated glioma cells, directly induce microglial cell migration and infiltration. 


\section{Discussion}

To the best of our knowledge, this study is the first to report the effect of necrosis on chemokine production and microglia infiltration mediated by GBM cells. We showed that NCs stimulate microglia migration/infiltration into GBM by upregulating CCL2/ MCP-1 and CCL20/MIP-3 $\alpha$ expression in glioblastoma cells (Fig. 7). Using the human GBM cell line and human microglia cell line, we demonstrated that the presence of NCs promoted the migration/infiltration of microglia, and that GBM cells exposed to NCs further enhanced the migration and infiltration of HMO6 microglial cells. Treatment of NCs induced the mRNA and protein expression of chemokines such as CCL2/MCP1 and CCL20/MIP-3 $\alpha$ in CRTMG cells. In particular, CCL2/

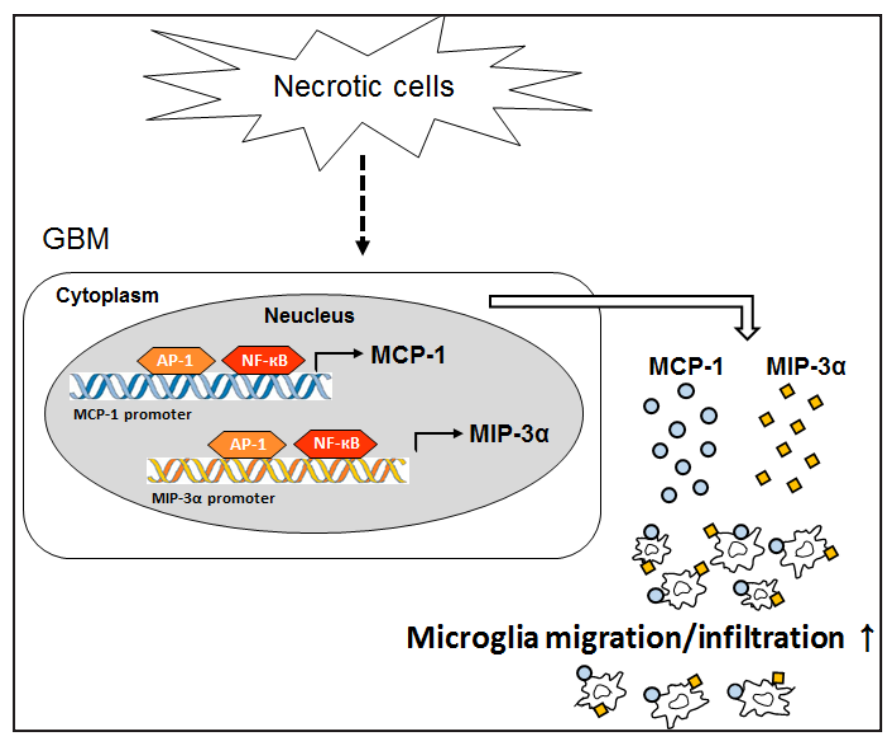

Fig. 7. A proposed model for glioblastoma microenvironment. Exposure to necrotic cells (NC) activates AP-1 and NF- $\kappa$ B transcription factors and their binding to the CCL2/MCP-1 and CCL20/MIP-3 $\alpha$ promoters, leading to enhanced CCL2/MCP-1 and CCL20/MIP-3 $\alpha$ production and secretion in glioblastoma cells. As a result, an increase in CCL2/MCP-1 and CCL20/MIP-3 $\alpha$ secretion from NC-exposed glioblastoma promotes migration/infiltration of microglial cells within glioblastoma. MCP-1 and CCL20/MIP-3 $\alpha$ were significantly increased in NC-treated CRT-MG and U87-MG cells. NCs induced DNA binding of the transcription factors NF- $\mathrm{KB}$ and AP- 1 to the CCL2/MCP-1 and CCL20/MIP-3 $\alpha$ promoters, leading to increased CCL2/MCP-1 and CCL20/MIP-3 $\alpha$ mRNA and protein expression.

GBM is characterized by the presence of necrosis, which has been considered a key diagnostic criterion for GBM and is correlated with poorer patient outcome [7]. Several studies have shown that tumor necrosis is associated with a poorer prognosis, advanced malignant progression, and decreased survival rate. In endometrial cancer, tumor necrosis is related to enhanced angiogenesis via increased expression of angiogenic markers such as Vascular Endothelial Growth Factor A (VEGF-A), VEGF-C, VEGF-D, and Fibroglast Growth Factor ( $\beta F G F)$ [34]. In renal cell carcinoma, the extent of necrosis is correlated with higher grade and greater tumor size [35]. During necrosis, cell death leads to the release of cellular contents into the extracellular environment, which in turn promotes cell growth, cancer progression, and tumor-infiltrating leukocyte recruitment. For example, high-mobility group box 1 protein, a proinflammatory mediator that distinguishes necrosis from apoptosis, is known to promote the recruitment of inflammatory cells [36]. Our results demonstrate that NC-diluted medium (NC-only) could increase the migration/infiltration of microglial cells in a NC-number-dependent manner (Fig. 1). However, the degree of migration/infiltration of HMO6 cells was further enhanced when CRT-MG cells were exposed to and stimulated by NCs, suggesting that CRT-MG cells exposed to NCs enhance the migration and infiltration of HMO6 microglial cells. These results indicate that the interaction between necrosis and glioblastoma cells greatly contributes to microglia infiltration within GBM.

In this study, we showed several chemokines produced by NC-treated GBM by performing microarray and chemokine array, suggesting that these chemokines released from cancer cells could promote and facilitate the migration/infiltration of microglia cells. The inflammatory microenvironment of a tumor contains a significant number of host leukocytes, including macrophages, dendritic cells, and lymphocytes [37]. Of these, TAMs are a major component of the infiltrate in tumors, and accumulating evidence demonstrates that increased density 
of macrophages is associated with tumor growth and metastasis in human malignancies, including breast cancer, gastric cancer, and GBM $[9,14,15,38]$. TAMs are known to be recruited by chemokines, which are secreted from tumor tissues. The levels of CCL2/MCP-1, CCL3/MIP-1 $\alpha$, and CCL5/RANTES were higher in non-small cell lung cancer (NSCLC) tumor tissues than in normal lung tissues, correlated with the degree of infiltrated macrophages within tumor tissues [39]. In colorectal cancers, the infiltration of macrophages is positively correlated with the level of CCL2/MCP-1 expression [16]. Previous studies have shown that the chemokines, such as CX3CL1/fractalkine and CCL2/MCP-1, and their receptors play important roles in the infiltration of microglia/macrophages in GBM [17, 28, 29]. Our results demonstrate that both CCL2/MCP-1 and CCL20/MIP-3 $\alpha$ increased in NC-treated glioma cells in a NC ratio-dependent manner (Figs. 3b and 3c) and addition of neutralizing CCL2/MCP1 and CCL20/MIP-3 $\alpha$ antibodies to NC-GCM significantly reduced migration/infiltration of microglial cells (Figs. 6d and 6f), indicating that these chemokines contribute to an NC-ratiodependent increase in the migration/infiltration of microglial cells.

In human glomerular endothelial cells, TNF- $\alpha$ increased CCL2/MCP-1 expression via the cooperative action of NF- $\kappa \mathrm{B}$ and AP-1 [40]. Interaction between CCL20 and CCR6 that promotes the invasion and migration of thyroid cancer cells is associated with NF- $\kappa \mathrm{B}$ activation [25]. Prolactin induces CCL20/MIP-3 $\alpha$ production through AP-1 activation in keratinocytes [41]. We found putative NF- $\kappa \mathrm{B}$ and AP-1 transcription factor-binding sites in the CCL2/MCP-1 and CCL20/MIP-3 $\alpha$ promoters (Figs. 4a and 4b). Our results also show that CCL2/MCP-1 and CCL20/MIP-3 $\alpha$ production in NC-treated GBM is mediated by NF- $\kappa B$ and AP-1 activation. In the current study, it was observed that anti-p50 antibody supershifted or weakened the DNA-protein complex, whereas anti-p65 antibody did not. These results indicate the specific interaction between the p50 subunit and NF- $\kappa B$ element of CCL2/MCP-1 and CCL20/MIP-3 $\alpha$.

In summary, we showed that NCs stimulate chemokine production in GBM cells, leading to significant promotion of microglial infiltration into GBM. Remarkably, although NCs alone influenced the migration/infiltration of microglia cells, glioblastoma cells that were exposed to NCs stimulated enhanced microglia migration/infiltration. Our results suggest that chemokines produced by NC-exposed GBM cells promote and facilitate the migration and infiltration of microglia, thus providing a better understanding of the effect of necrosis on microglia infiltration in GBM.

\section{Acknowledgements}

This work was supported by a National Research Foundation of Korea (NRF) grant funded by the Korean government (MSIP) Grant 2010-0027945 and by NRF-2016R1A2B4016376.

\section{Disclosure Statement}

The authors declare no conflict of interests.

\section{References}

1 Hung FH, Chiu HW: Cancer subtype prediction from a pathway-level perspective by using a support vector machine based on integrated gene expression and protein network. Comput Methods Programs Biomed 2017;141:27-34.

-2 Furnari FB, Fenton T, Bachoo RM, Mukasa A, Stommel JM, Stegh A, Hahn WC, Ligon KL, Louis DN, Brennan C, Chin L, DePinho RA, Cavenee WK: Malignant astrocytic glioma: genetics, biology, and paths to treatment. Genes Dev 2007;21:2683-2710. 


\section{Cellular Physiology Cell Physiol Biochem 2018;48:1332-1346

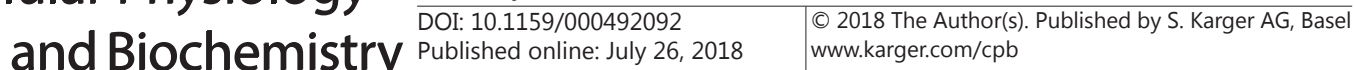

Jung et al.: Necrosis Induces Microglia Infiltration in Glioblastoma

3 Raza SM, Lang FF, Aggarwal BB, Fuller GN, Wildrick DM, Sawaya R: Necrosis and glioblastoma: a friend or a foe? A review and a hypothesis. Neurosurgery 2002;51:2-12.

4 Narsia N, Ramagiri P, Ehrmann J, Kolar Z: Transcriptome analysis reveals distinct gene expression profiles in astrocytoma grades II-IV. Biomed Pap Med Fac Univ Palacky Olomouc Czech Repub 2017;161:261-271.

5 Simmons GW, Pong WW, Emnett RJ, White CR, Gianino SM, Rodriguez FJ, Gutmann DH: Neurofibromatosis-1 heterozygosity increases microglia in a spatially and temporally restricted pattern relevant to mouse optic glioma formation and growth. J Neuropathol Exp Neurol 2011;70:51-62.

6 Ahn SH, Park H, Ahn YH, Kim S, Cho MS, Kang JL, Choi YH: Necrotic cells influence migration and invasion of glioblastoma via NF-kappaB/AP-1-mediated IL-8 regulation. Sci Rep 2016;6:24552.

7 Barker FG, 2nd, Davis RL, Chang SM, Prados MD: Necrosis as a prognostic factor in glioblastoma multiforme. Cancer 1996;77:1161-1166.

-8 Li W, Graeber MB: The molecular profile of microglia under the influence of glioma. Neuro Oncol 2012;14:958-978.

-9 Cummings TJ, Hulette CM, Bigner SH, Riggins GJ, McLendon RE: Ham56-immunoreactive macrophages in untreated infiltrating gliomas. Arch Pathol Lab Med 2001;125:637-641.

-10 Santambrogio L, Belyanskaya SL, Fischer FR, Cipriani B, Brosnan CF, Ricciardi-Castagnoli P, Stern LJ, Strominger JL, Riese R: Developmental plasticity of CNS microglia. Proc Natl Acad Sci U S A 2001;98:62956300.

11 Hambardzumyan D, Gutmann DH, Kettenmann H: The role of microglia and macrophages in glioma maintenance and progression. Nat Neurosci 2016;19:20-27.

-12 Solinas G, Germano G, Mantovani A, Allavena P: Tumor-associated macrophages (TAM) as major players of the cancer-related inflammation. J Leukoc Biol 2009;86:1065-1073.

13 Susan F, Nephi S: Targeting endocannabinoid signaling in tumor-associated macrophages as treatment for glioblastoma multiforme. Wiley Interdisciplinary Reviews: Membrane Transport and Signaling 2014;3:3951.

14 Wesolowska A, Kwiatkowska A, Slomnicki L, Dembinski M, Master A, Sliwa M, Franciszkiewicz K, Chouaib S, Kaminska B: Microglia-derived TGF-beta as an important regulator of glioblastoma invasion--an inhibition of TGF-beta-dependent effects by shRNA against human TGF-beta type II receptor. Oncogene 2008;27:918930.

15 Fonseca AC, Romao L, Amaral RF, Assad Kahn S, Lobo D, Martins S, Marcondes de Souza J, Moura-Neto V, Lima FR: Microglial stress inducible protein 1 promotes proliferation and migration in human glioblastoma cells. Neuroscience 2012;200:130-141.

16 Bailey C, Negus R, Morris A, Ziprin P, Goldin R, Allavena P, Peck D, Darzi A: Chemokine expression is associated with the accumulation of tumour associated macrophages (TAMs) and progression in human colorectal cancer. Clin Exp Metastasis 2007;24:121-130.

17 Held-Feindt J, Hattermann K, Muerkoster SS, Wedderkopp H, Knerlich-Lukoschus F, Ungefroren H, Mehdorn HM, Mentlein R: CX3CR1 promotes recruitment of human glioma-infiltrating microglia/ macrophages (GIMs). Exp Cell Res 2010;316:1553-1566.

-18 Salazar N, Castellan M, Shirodkar SS, Lokeshwar BL: Chemokines and chemokine receptors as promoters of prostate cancer growth and progression. Crit Rev Eukaryot Gene Expr 2013;23:77-91.

19 Hesselgesser J, Horuk R: Chemokine and chemokine receptor expression in the central nervous system. J Neurovirol 1999;5:13-26.

20 Rotondi M, Chiovato L, Romagnani S, Serio M, Romagnani P: Role of chemokines in endocrine autoimmune diseases. Endocr Rev 2007;28:492-520.

21 Moser B, Wolf M, Walz A, Loetscher P: Chemokines: multiple levels of leukocyte migration control. Trends Immunol 2004;25:75-84.

22 Beppu M, Sawai S, Misawa S, Mori M, Ito S, Sogawa K, Nishimura M, Matsushita K, Nomura F, Kuwabara S: Serum cytokine and chemokine profiles in patients with juvenile muscular atrophy of distal upper extremity (Hirayama disease). J Neuroimmunol 2017;302:20-22.

-23 Cui X, Li Z, Gao J, Gao PJ, Ni YB, Zhu JY: Elevated CXCL1 increases hepatocellular carcinoma aggressiveness and is inhibited by miRNA-200a. Oncotarget 2016;7:65052-65066. 


\section{Cellular Physiology Cell Physiol Biochem 2018;48:1332-1346 \begin{tabular}{l|l} 
and Biochemistry Published onlıne: July 26, 2018 & $\begin{array}{l}\text { (c) } 2018 \text { The Author(s). Published by S. Karger AG, Basel } \\
\text { www.karger.com/cpb }\end{array}$ \\
\hline
\end{tabular}}

Jung et al.: Necrosis Induces Microglia Infiltration in Glioblastoma

24 Ueno T, Toi M, Saji H, Muta M, Bando H, Kuroi K, Koike M, Inadera H, Matsushima K: Significance of macrophage chemoattractant protein-1 in macrophage recruitment, angiogenesis, and survival in human breast cancer. Clin Cancer Res 2000;6:3282-3289.

25 Zeng W, Chang H, Ma M, Li Y: CCL20/CCR6 promotes the invasion and migration of thyroid cancer cells via NF-kappa B signaling-induced MMP-3 production. Exp Mol Pathol 2014;97:184-190.

-26 Maolake A, Izumi K, Shigehara K, Natsagdorj A, Iwamoto H, Kadomoto S, Takezawa Y, Machioka K, Narimoto K, Namiki M, Lin WJ, Wufuer G, Mizokami A: Tumor-associated macrophages promote prostate cancer migration through activation of the CCL22-CCR4 axis. Oncotarget 2017;8:9739-9751.

-27 Ben-Baruch A: The multifaceted roles of chemokines in malignancy. Cancer Metastasis Rev 2006;25:357371.

28 Leung SY, Wong MP, Chung LP, Chan AS, Yuen ST: Monocyte chemoattractant protein-1 expression and macrophage infiltration in gliomas. Acta Neuropathol 1997;93:518-527.

-29 Zhu X, Fujita M, Snyder LA, Okada H: Systemic delivery of neutralizing antibody targeting CCL2 for glioma therapy. J Neurooncol 2011;104:83-92.

-30 Ma Z, Qin H, Benveniste EN: Transcriptional suppression of matrix metalloproteinase-9 gene expression by IFN-gamma and IFN-beta: critical role of STAT-1alpha. J Immunol 2001;167:5150-5159.

-31 Nagai A, Nakagawa E, Hatori K, Choi HB, McLarnon JG, Lee MA, Kim SU: Generation and characterization of immortalized human microglial cell lines: expression of cytokines and chemokines. Neurobiol Dis 2001;8:1057-1068.

-32 Yim N, Ryu SW, Choi K, Lee KR, Lee S, Choi H, Kim J, Shaker MR, Sun W, Park JH, Kim D, Heo WD, Choi C: Exosome engineering for efficient intracellular delivery of soluble proteins using optically reversible protein-protein interaction module. Nat Commun 2016;7:12277.

-33 Flynn G, Maru S, Loughlin J, Romero IA, Male D: Regulation of chemokine receptor expression in human microglia and astrocytes. J Neuroimmunol 2003;136:84-93.

-34 Bredholt G, Mannelqvist M, Stefansson IM, Birkeland E, Bo TH, Oyan AM, Trovik J, Kalland KH, Jonassen I, Salvesen HB, Wik E, Akslen LA: Tumor necrosis is an important hallmark of aggressive endometrial cancer and associates with hypoxia, angiogenesis and inflammation responses. Oncotarget 2015;6:39676-39691.

-35 Lam JS, Shvarts O, Said JW, Pantuck AJ, Seligson DB, Aldridge ME, Bui MH, Liu X, Horvath S, Figlin RA, Belldegrun AS: Clinicopathologic and molecular correlations of necrosis in the primary tumor of patients with renal cell carcinoma. Cancer 2005;103:2517-2525.

36 Vakkila J, Lotze MT: Inflammation and necrosis promote tumour growth. Nat Rev Immunol 2004;4:641648.

37 Balkwill F, Mantovani A: Inflammation and cancer: back to Virchow? Lancet 2001;357:539-545.

38 Chen Y, Zhang S, Wang Q, Zhang X: Tumor-recruited M2 macrophages promote gastric and breast cancer metastasis via M2 macrophage-secreted CHI3L1 protein. J Hematol Oncol 2017;10:36.

-39 Arenberg DA, Keane MP, DiGiovine B, Kunkel SL, Strom SR, Burdick MD, Iannettoni MD, Strieter RM: Macrophage infiltration in human non-small-cell lung cancer: the role of CC chemokines. Cancer Immunol Immunother 2000;49:63-70.

-40 Park SK, Yang WS, Han NJ, Lee SK, Ahn H, Lee IK, Park JY, Lee KU, Lee JD: Dexamethasone regulates AP-1 to repress TNF-alpha induced MCP-1 production in human glomerular endothelial cells. Nephrol Dial Transplant 2004;19:312-319.

-41 Kanda N, Shibata S, Tada Y, Nashiro K, Tamaki K, Watanabe S: Prolactin enhances basal and IL-17-induced CCL20 production by human keratinocytes. Eur J Immunol 2009;39:996-1006. 